\title{
Timber Characterisation Using A Non-Invasive TDR Sensor
}

\author{
M.Hagedorn, I. G. Platt and I. M. Woodhead \\ Lincoln Ventures Ltd. \\ Christchurch \\ New Zealand \\ hagedorm@lvl.co.nz
}

\begin{abstract}
This paper utilizes the technique of Time Domain Reflectometry (TDR) to aid in the characterization of timber. The electric field of TDR sensor is very sensitive to moisture trapped within the timber and can thus be used to infer anomalies in the grain down to a depth of approximately $10 \mathrm{~cm}$ below its surface. This novel application of non-invasive TDR indicates the presence of timber characteristics such as size and frequency of knots, splits and decay by a time series analysis of the propagation delays as the TDR sensor probe is moved along the timber surface.
\end{abstract}

\section{INTRODUCTION}

Timber condition estimates have always been an important part of the timber industries strategy for dispatching the appropriate product to its customer base. Sensors are increasingly finding their way into this process [1] to fulfill requirements for rapid inline measurement and to meet industry demands for enhanced inspection (i.e. sub-surface, or throughout the volume). Currently, inspection of timber for particular down-grading features such as knots, splits and decay is achieved by manual or automated visual inspection of the timber surface. There is increasing demand by the industry for better techniques that will enable early upgrade or downgrade of timber with subsurface defects that may not be visually apparent until further processing by the customer. Better early prediction of subsurface defects will thus enhance the value of the timber and avoid costly rejection of timber by the customer.

The non-invasive Time Domain Reflectometry (TDR) approach offers several advantages over other methods including (1) non destructive evaluation, (2) very rapid measurement cycles, (3) the possibility of a very compact and robust installation and (4) accurate measurements which are largely unaffected by the harsh timber mill environment.

This paper describes an experiment designed to test the viability of using the TDR methodology in detecting defects, such as knots and cracks in timber samples.

\section{EXPERIMENTAL SETUP}

The non-invasive TDR technique used here utilizes a parallel transmission line to detect changes in the pulse velocity and amplitude as its transverse field interacts with the induced polarization field of the sample's molecules [2] [3]. Since the relative permittivity of water is high $(\sim 80)$ compared to other materials such as wood, the measurement is sensitive to variations in timber moisture, and also in density variations mainly due to differences in moisture accumulation. A schematic of the set up is shown in Fig. 1. Measurements were made with a Hewlett Packard (Agilent) HP54121T digitising oscilloscope with integral TDR step generator. The parallel transmission line was connected to the HP54121T through a Guanella-type balun constructed in a similar manner to the 1:4 balun described by [4] but used a $3.5 \mathrm{~mm}$ diameter, grade S3 ferrite toroid with three turns of $0.125 \mathrm{~mm}$ enamelled wire. The transmission line was constructed from 8 $\mathrm{mm}$ diameter $500 \mathrm{~mm}$ long brass rods spaced $75 \mathrm{~mm}$ apart. Although stainless steel rods have been used for conventional TDR measurements, they resulted in an approximately $100 \mathrm{ps}$ degradation in the risetime of the voltage step from the balun, leading us to use a lower loss material. The brass rods had a negligible effect on the risetime.

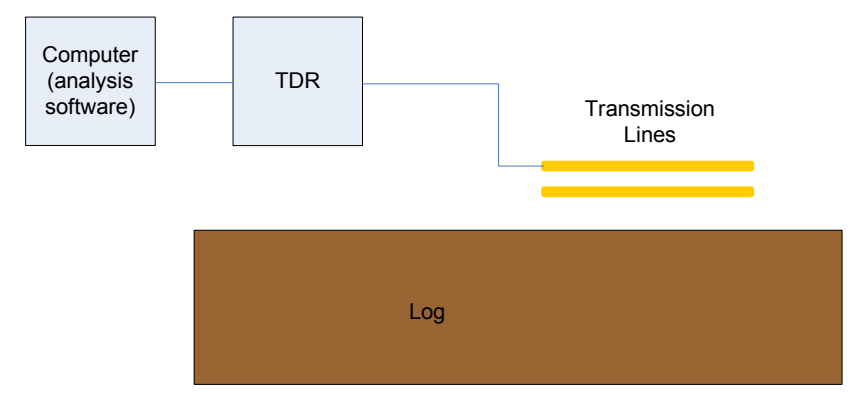

Figure 1 Schematic of the setup utilizing parallel transmission lines for a Time Domain Reflectometry (TDR) detection of timber anomalies. 
The sample of untreated Oregon pine used for the test described here is shown in Fig. 2a. The sample has a number of clearly visible knots, one extending through the sample to be visible on the opposite surface. For each side, the transmission lines were moved at $1 \mathrm{~cm}$ intervals along both the width and the length of the sample, taking time of flight and amplitude measurements of the pulse. The mid-point between the transmission lines is used as the measurement reference and to avoid large edge effects both lines must be over the sample. Since the lines are $7.5 \mathrm{~cm}$ apart there is a $3.75 \mathrm{~cm}$ edge around the sample that is not measured. Further, because the induced field extends beyond the region between the lines, some effect due to the edge between the sample and the air will be observed near the upper and lower extremities of the measurements.

\section{RESULTS}

Fig. 2 shows the difference in TDR pulse amplitude from the overall mean, at each $1 \mathrm{~cm}$ location, when the transmission lines are placed across the sample width. The mean waveform amplitude at a specific time is calculated from all TDR measurements at that time.

Before discussing the correlation with the timber features several aspects of Fig. 2 require clarification. Firstly the changes in amplitude are the result of both direct signal loss in the material and a shift due to time delay. For example the large amplitude change toward the end of the pulse is due to the shift in a steep reflection gradient along the time axis; the delay due to the change in pulse velocity when passing over differences in permittivity. This effect is cumulative and is most obvious near the end of the transmission lines, away from the sample. The peaks in amplitude change before this are largely due to loss and occur at the position where the discontinuity exists. The gradient due to reflection is most evident in Fig. 3 where a single TDR measurement with the centre of the transmission lines at $0.1 \mathrm{~m}$ is shown.

Fig. 2 can thus be viewed as representing two effects, both a good indication of the presence of irregularities in the sample, one providing position and the other potentially (though not investigated here) the change in permittivity.

Also shown in Fig. 2 are the boundaries of the sample, those vertically (along the length of the sample) determined in the time domain by shorting the lines at these positions and those horizontally by the transmission line centre geometry described in Section II.

Comparing the photo of the sample and the reconstruction plot in Fig 2 it is clear that the two major knot regions labeled $\mathrm{A}$ and $\mathrm{B}$ have been detected by the TDR process. Knot $\mathrm{C}$ is obviously out of the observation region. Close inspection of knot $\mathrm{B}$ indicates that it is closer to the right boundary than expected and is likely due to a combination of effects including, (1) misplacement of the boundary due to incorrect interpretation of velocity components across the sample (currently under investigation) and more importantly (2) the knot labeled B moves toward the right boundary with depth through the sample. Since the field of the TDR penetrates well into the sample $(\sim 0.1 \mathrm{~m})$ the plot in Fig. 2 is actually the

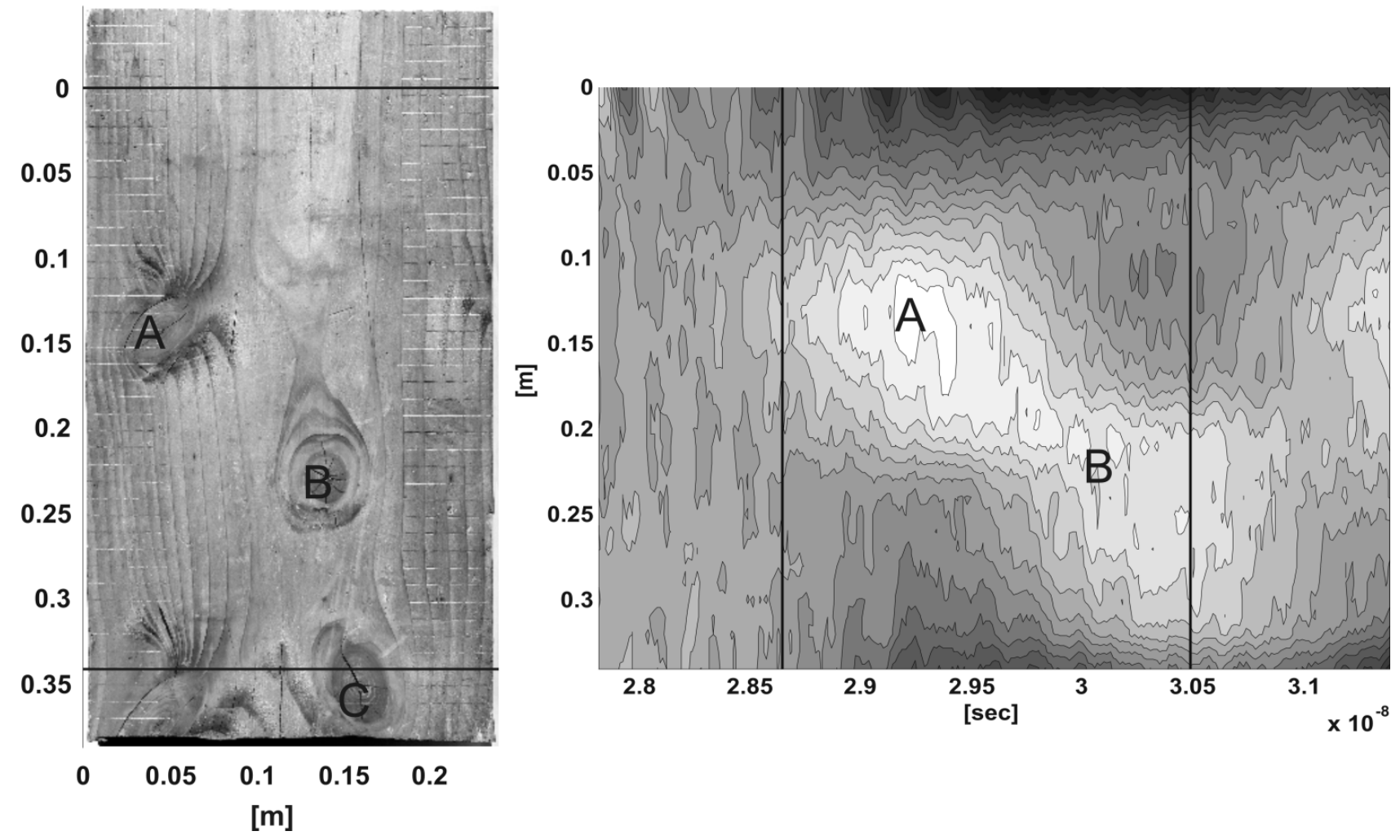

Figure 2 Photo of the timber sample and a plot of the reconstructed TDR measurements. The two knots labeled A and B are evident in the via changes in the amplitude over their locations. The measurement region is between the two horizontal lines on the photo (to to which the transmission lines are parallel) while the interpreted position of the sample edges (in width) are shown by the vertical lines on the plot. 


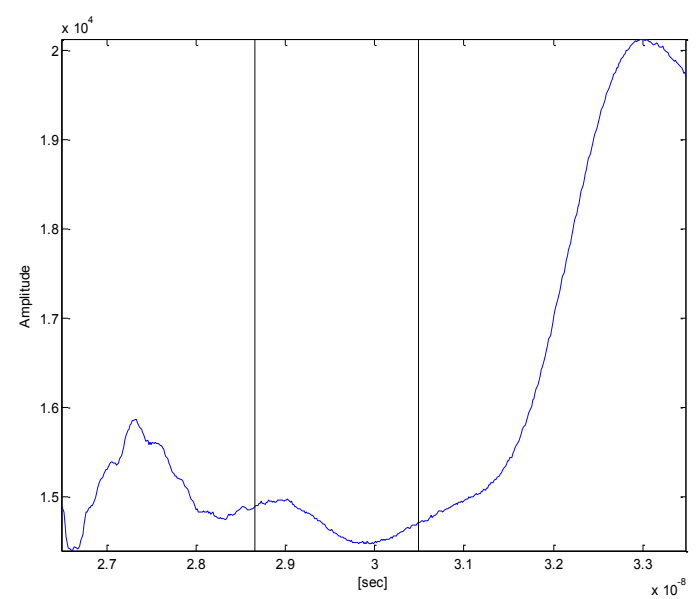

Figure 3 A single TDR measurement taken with the transmission lines centred at $0.1 \mathrm{~m}$ (along side of Fig. 2). The sample position is between the two vertical lines.

composition of signal losses and time delays throughout the samples depth, in this case moving the focus toward the right boundary.

Figs. 4 and 5 show the TDR results for the reverse side of the same sample. The orientation of the transmission line scans of Fig. 4 are the same as those used in the previous example (i.e. the transmission lines are parallel to the bottom edge) while those of Fig. 4 are at right angles to the Fig. 2 scans. The obvious knot, actually the penetrated knot B of Fig. 2 , is well defined by both transmission line geometries and well correlated in position. The plot in Figure 4 indicates a region of permittivity difference in the top right corner not associated with any visible feature. Whether this is a processing artifact or a real change in moisture content not visible on the surface is unknown and is the subject of further investigation.

All plots show a tendency for features to be elongated in the direction of the transmission lines. This is largely due to the cumulative time delay differences the feature introduces, resulting in smearing of the signal around its edges. Such effects can be accounted for once the velocity field (i.e. propagation time across segments of the sample) is analyzed in more detail and this is also the subject of future work.

\section{CONCLUSION AND DISCUSION}

The results of the experiment detailed in this paper show that the TDR measurements can accurately determine the location of anomalies (in this case knots) in a sample of wood. Further, since the technique is sensitive to structure at and below the surface of the sample, the detection cross section is much larger than that afforded by a visual inspection, resulting in a much improved condition assessment. Although not yet calibrated to do so the above results also indicate that this technique may be used to estimate the strength of an anomaly.

The results presented here are derived from a straightforward application of the TDR system to the sample using just the amplitude differences. As mentioned in Section III a large part of the information, that due to time delay measurement, has not been utilized. The time delay can be simply used to increase the resolution of the measurements, or more usefully, to reconstruct the permittivity properties of the sample via tomographic imaging. For this application the transmission line scans are performed to provide a parallel projection for each nominated angle around the sample. The

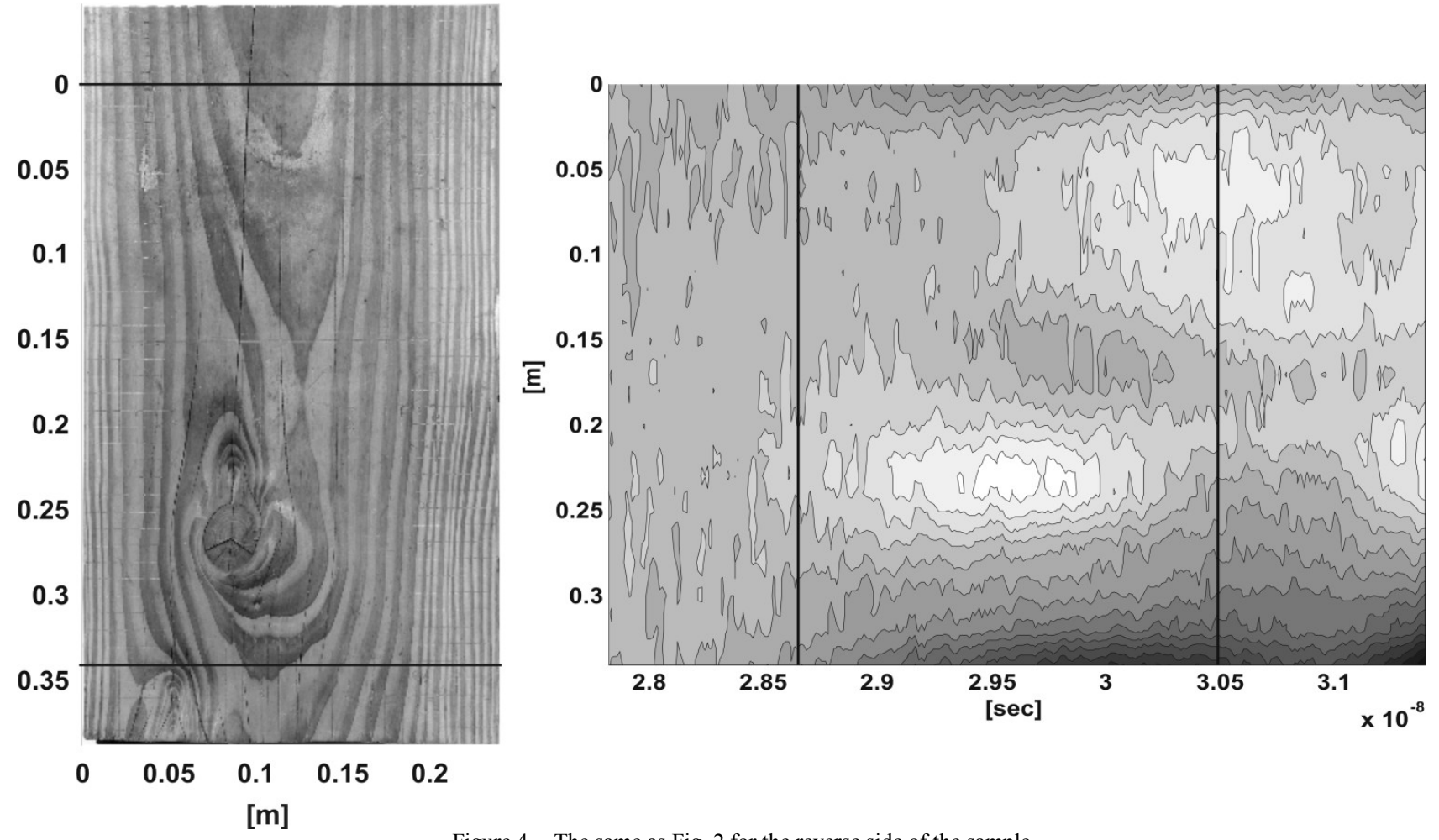

Figure 4 The same as Fig. 2 for the reverse side of the sample. 


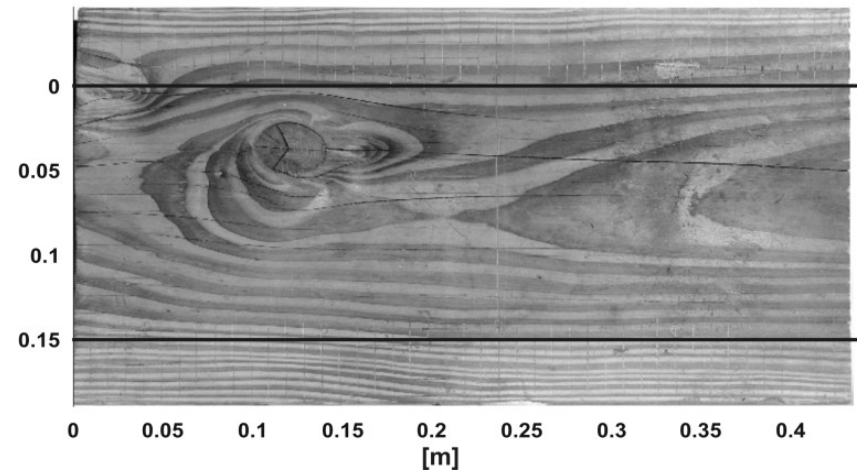

Figure 5 The reverse side of the sample with the parallel transmission lines scanned at 90 o to those in Fig. 4.

number of angles chosen to be used will determine the resolution of the reconstructed velocity field and thus by the inversion defined in [3] the permittivity profile across the sample. Relating the permittivity to the moisture content [5] provides a useful measure for many applications and sample types. This application of temporal data is the subject of current research.

Future work will also be directed towards improving the design of the transmission lines to better match the operational setup of the particular application. Also application specific is work to characterize the TDR signatures into a reference base so that specific types of features can be recognized along with an estimate of their properties. While the example here is for the timber industry, a number of other applications are also being considered including, (1) moisture content variations in

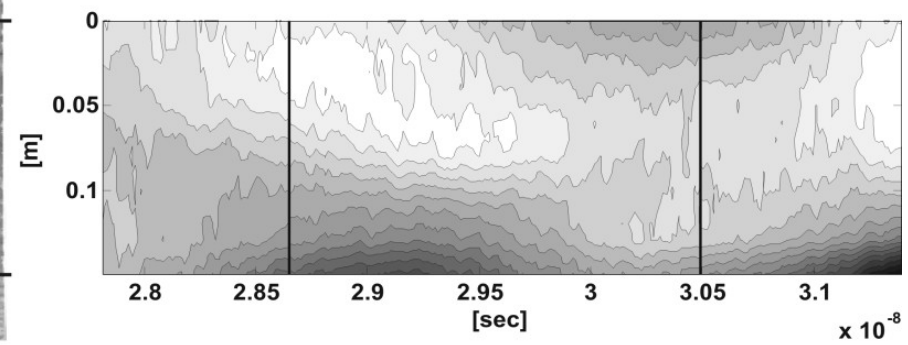

concrete that may be tuned to indicate curing anomalies, cracks and plasticity etc. and (2) road construction integrity.

\section{REFERENCES}

[1] Bucur V. "Techniques for high resolution imaging of wood structure: a review", Meas. Sci. and Tech., 14, 2003.

[2] Woodhead, I.M., Buchan, G.D. and Kulasiri, D. "Pseudo-3-D moment method for rapid calculation of electric field distribution in a low-loss inhomogeneous dielectric", IEEE Trans. Antenna and Propagat., vol. 35, pp. 1117-1122, 2001.

[3] Platt, I.G. and Woodhead, I.M., "A 1D inversion for non-invasive time domain reflectometry”, Meas. Sci. Tech., vol. 19, pp 055708, 2008.

[4] Spaans E J A and Baker J M., Simple Baluns in Parallel Probes for Time Domain Reflectometry, Soil Science Society American Journal, vol. Vol. 57, pp. 668-673, 1993

[5] Topp, G. C. and Davis, J. L. and Annan, A. P., A Electromagnetic Determination of Soil Water Content Using TDR: I. Applications to Wetting Fronts and Steep Gradients, Soil Sci Soc Am J, vol. 46, pp 672-678, 1982. 